Please do not remove this page

RMIT

UNIVERSITY

\title{
Using information foraging theory to understand search behavior in different environments
}

Ong, Kevin

https://researchrepository.rmit.edu.au/esploro/outputs/9921863309701341/filesAndLinks?institution=61RMIT_INST\&index=null

Ong, K. (2017). Using information foraging theory to understand search behavior in different environments. Proceedings of the 2017 Conference on Conference Human Information Interaction and Retrieval (CHIIR 2017), 411-413.

https://researchrepository.rmit.edu.au/discovery/fulldisplay/alma9921863309701341/61RMIT_INST:Resea rchRepository

Document Version: Published Version

Repository homepage: https://researchrepository.rmit.edu.au

(C) 2017 Copyright held by the owner/author(s).

Downloaded On 2023/04/26 22:50:43 +1000 
Thank you for downloading this document from the RMIT Research Repository.

The RMIT Research Repository is an open access database showcasing the research outputs of RMIT University researchers.

RMIT Research Repository: http://researchbank.rmit.edu.au/

\section{Citation:}

Ong, K 2017, 'Using information foraging theory to understand search behavior in different environments', in Proceedings of the 2017 Conference on Conference Human Information Interaction and Retrieval (CHIIR 2017), Oslo, Norway, 7-11 March 2017, pp. 411-413.

See this record in the RMIT Research Repository at:

http://researchbank.rmit.edu.au/view/rmit:48575

Version: Published Version

Copyright Statement:

(C) ( 2017 Copyright held by the owner/author(s).

\section{Link to Published Version:}

https://dl.acm.org/citation.cfm?id=3022173 


\title{
Using Information Foraging Theory to Understand Search Behavior in Different Environments
}

\author{
Kevin Ong \\ RMIT University \\ Melbourne, Australia \\ kevin.ong@rmit.edu.au
}

\begin{abstract}
The aim of this research proposal is to understand the extent in which Information Scent, part of Information Foraging Theory, influence the perception of relevance of retrieved search engine result pages (SERPs) in different environments (desktop and mobile). Firstly, we planned to investigate how previously found correlations between information scent and search behavior on desktop SERPs may also applicable on mobile. Secondly, we investigate if the differences in search behavior are also caused by SERP visibility and/or physical input behavior in different environments. Lastly, we would like to observe the extent in which changing snippet length can affect web search behavior. The perception of relevance on SERPs might be similar in both environments because the arrangement of SERPs on both mobile and desktop are identical; or different because the visibility of search results is less on mobile compared to desktop, due to limited screen sizes. We wanted to study the extent of which the physical limitations might significantly impact search behavior in different environments due to expected "hidden scent". We conducted two user studies to observe these phenomenon and seek to understand search behavior by manipulating the SERPs presented to users. We observed both similarities and differences in web search behavior between the environments. On the desktop, increasing information scent led to lower positions of search results saved as relevant and lower positions of mouse hover. However, on mobile, increasing relevant search results beyond the initial screen size, reduced the number of documents examined.
\end{abstract}

\section{Categories and Subject Descriptors}

H.3.3 [Information Storage and Retrieval]: Information Search and Retrieval-search process; selection process

\section{Keywords}

Search Behavior; Search Strategies; Query Reformulation;

Permission to make digital or hard copies of part or all of this work for personal or classroom use is granted without fee provided that copies are not made or distributed for profit or commercial advantage and that copies bear this notice and the full citation on the first page. Copyrights for third-party components of this work must be honored. For all other uses, contact the owner/author(s).

CHIIR '17 March 07-11, 2017, Oslo, Norway

(C) 2017 Copyright held by the owner/author(s).

ACM ISBN 978-1-4503-4677-1/17/03.

DOI: http://dx.doi.org/10.1145/3020165.3022173
Information Foraging Theory

\section{INTRODUCTION}

Information Foraging Theory [4] is a theory that seeks to predict how rational information seekers behave when finding relevant information. They seek to maximize information gain by minimizing the cost of information seeking. Information Scent, part of Information Foraging Theory, has been observed to alter web search behavior when SERPs were intentionally manipulated. Past work [8] found that stronger information scent, increased document examinations and clicked deeper on the search results. We seek to understand if this behavior is also consistent on mobile.

\subsection{Motivation}

The rise of mobile search $^{1}$ meant that, unlike traditional desktop search, perception of relevance of search results will be constrained. Regardless of the direction that modern mobile engine is taking, understanding how "hidden scent" can affect search behavior is worth investigating because if the expected "hidden scent" plays a role in searching, we may need to evaluate searching in different environments differently.

While mobile phones typically have the smallest screens compared to tablets or desktops, they have the highest mobility and timely access to up-to-date information. These differences suggest different devices types may satisfy different information needs [5]. However, there are reasons to expect similarities as well. They are similar because they all provide access to roughly the same Internet sources with similar amounts of information. We are motivated to use Information Foraging Theory to understand how the extent in which Information Scent affect search behavior in different environments.

\section{RESEARCH QUESTIONS}

The main research questions are as follows:

Research Question 1 (RQ1): To what degree, can mobile and desktop web search behavior be explained by Information Scent Theory?

$\mathrm{Wu}$ et al. [9] found that while interacting with SERPs more relevant search results, searchers examined more documents and clicked deeper in the search result list; also found searchers

\footnotetext{
${ }^{1}$ http://selnd.com/2aJcPv1

${ }^{2}$ relevant search results not immediately seen
} 
to more likely abandon queries, if relevant results are only located lower on the SERPs. We want to study if the phenomenon would happen regardless of the environments. Therefore, we gave the searchers same topics for both environments and observe if they would behave differently. Addressing this question can enable us to extend our knowledge on the applicability of Information Foraging Theory in different environments.

Research Question 2 (RQ2): To what degree, does mobile and desktop query reformulations affected by Information Scent and typing fluency [3]?

We seek to address if users' propensity for query reformulation is influenced by Information Scent and/or typing fluency. There are two distinct forms of search strategies [7]. We want to investigate how different environments (between desktops and mobiles) may reflect changes in search strategies. Addressing this question will enable a better understanding of information seeking behavior that is influenced by user's physical characteristics. By extending this work to individually measure typing characteristics of each query issue by users, we can test the validity of using typing characteristics to categorize users into task familiarity and topic expertise. This can potentially be extended to voice search and visually handicapped information seekers.

Research Question 3 (RQ3): To what degree, does snippet length affect search behavior in different environments? We will like to explore the impact that different snippet length have on user's web search behavior. Previously, Cutrell and Guan [1] have identified that lengthening snippets aided users in information-seeking tasks. However, Song et al.[5] have observed that navigational tasks on portable devices (tablets and smartphones) are diminishing due to availability of app store on smartphones. By focusing our attention on information-seeking tasks, how much will user information seeking behavior change, due to different snippet lengths. To this aim, we will also investigate how different snippet lengths can affect search behavior across different environments.

\section{METHODOLOGY}

In this section, we will demonstrate how the research questions can be addressed. The methodology is ongoing for RQ1 and RQ2 and planned for RQ3.

RQ1 \& 2: Comparative user studies: In the first stage, we conducted two user studies to compare users on mobile phones and desktops using previous research framework [8]. We created both mobile and desktop search interfaces to capture the data from the user studies. By conducting user studies of our own and comparing against previous study, we can validate previous study's results and adopt appropriate measures to conduct further experiments. We split both user studies into 2 groups: Information Scent Level (ISL) and Information Scent Pattern (ISP). ISL refers to increasing level of topically relevant search results on the SERPs while ISP refers to different distribution of four topically relevant search results on the SERPs. There are two views on relevance, topicality or user-utility [6]. We estimated user utility by taking the top queries for the topics from previous experiment [8] to construct our SERPs before the experiments. The SERPs were constructed based on concepts that were relevant to the topic questions. Nonrelevant search results were included to vary the level of information scent. We acknowledged users might still issue queries that was significantly different from our pool. Therefore, they were instructed to find topically relevant results after their searches. We also recorded typing characteristics for each query that every user issued. They can then potentially be used to build user profiles on search experiences and task familiarity.

RQ3: Crowd experiment: To identify the effects of search snippet length on information needs, we plan to do the following experiments:

Create a desktop experiment with various length of search snippets with prefixed tasks: Using TREC collections to build tasks and relevant results with varying length of search snippets and conduct the experiment on crowd-sourced workers. Create a simulated mobile and tablet experiment with varying search snippet length with prefixed tasks: Using previous experiment resources, we will run our experiments on simulated displays on crowd-sourced workers. The displays will be simulated by predefining screen size on desktopsized screens to smaller dimensions to fit typical tablets and smartphone sizes.

\section{PROGRESS}

We have concluded the user studies and are currently analyzing the results for RQ1.

\subsection{Measurements}

Below is a subset of the measurements taken from the user studies:

- Time: Time spent examining search results for the first query per task

- NumPage: Number of SERP paginations for each task.

- NumExam: Number of documents examined for each search result set.

- DRS: Deepest rank scrolled into view. Lowest search result position that becomes visible in the viewable area during a search. If the participant paginate to second page, search depth ranges from $11-20$.

- DRH: Deepest rank hover (for desktop experiment). Lowest search result position that the mouse hovered over for at least 0.5 seconds. If users did not hover the mouse at all, the value will be 0 .

- ROA: Rate Of Abandonment, the rate of not clicking or saving any document as relevant after the initial query submission for each task.

- DRT: Deepest position on the first SERP saved as relevant by the users.

- TTotal: Total number of search results on the first SERP saved as relevant per task per participant.

- TRele: Total number of relevant search results on the first SERP (according to ISL/ISP conditions) saved by the users.

On Tables 1 and 2, Information Scent Level - Low (ILL), Information Scent Level - Medium (ILM) and Information Scent Level - High (ILH) referred to the number of topically relevant [6] search results presented to the users, arranged in ascending level of information scent from 1 to 3 to 5 from the top position on the SERPs. Details of the distribution can be found in previous work [8]. It shows that both on desktop and mobile, users tended to mark more documents (as well as more relevant documents) as relevant as ISL increased. Users also looked at more results (DRS) when more relevant 
Table 1: Increasing Information Scent Level (ISL) conditions

\begin{tabular}{|l|c|c|c|}
\hline \multicolumn{1}{|c|}{ ISL } & \multicolumn{3}{c|}{ Desktop } \\
\hline Measures & ILL & ILM & ILH \\
\hline \multirow{2}{*}{ Time } & 47.92 & 57.37 & $\mathbf{6 2 . 9 4}$ \\
& $(28.19)$ & $(38.11)$ & $\mathbf{( 3 9 . 8 5 )}$ \\
\hline NumPage & $.31(.47)$ & $.46(.51)$ & $\mathbf{. 4 9}(.51)$ \\
\hline NumExam & $.39(.49)$ & $.40(.81)$ & $\mathbf{. 6 9}(\mathbf{1 . 1 6}) *$ \\
\hline DRS & $12.44(5.18) *$ & $14.17(5.38) *$ & $\mathbf{1 4 . 3 4 ( 5 . 4 1 ) *}$ \\
\hline DRH & $6.11(7.04)$ & $7.89(6.85)$ & $\mathbf{8 . 6 4}(\mathbf{6 . 9 2})$ \\
\hline ROA & $39 \%$ & $23 \%$ & $\mathbf{1 7 \%}$ \\
\hline DRT & $.53(.56) * *$ & $1.86(1.44)$ & $\mathbf{3 . 1 7}(\mathbf{2 . 5 5})$ \\
\hline TTotal & $.53(.56) *$ & $1.60(1.33)$ & $\mathbf{2 . 3 7}(\mathbf{1 . 8 8})$ \\
\hline TRele & $.50(.51)$ & $1.49(1.09)$ & $\mathbf{2 . 2 6}(\mathbf{1 . 8 0})$ \\
\hline
\end{tabular}

\begin{tabular}{|c|c|c|}
\hline \multicolumn{3}{|c|}{ Mobile } \\
\hline ILL & ILM & ILH \\
\hline 43.42 & $\mathbf{6 1 . 6 1}$ & 53.39 \\
$(21.00)$ & $\mathbf{( 4 3 . 8 1 )}$ & $(26.20)$ \\
\hline $.28(.45)$ & $\mathbf{. 3 1}(.47)$ & $\mathbf{. 3 1}(.47)$ \\
\hline $.19(.40)$ & $\mathbf{. 5 6}(.91)$ & $.31(.92) *$ \\
\hline $10.83(5.60) *$ & $11.17(5.74) *$ & $\mathbf{1 1 . 6 9 ( 5 . 1 9 ) *}$ \\
\hline- & - & - \\
\hline $\mathbf{2 8 \%}$ & $11 \%$ & $11 \%$ \\
\hline $1.42(2.13) * *$ & $2.42(1.65)$ & $\mathbf{3 . 6 9}(\mathbf{2 . 4 5})$ \\
\hline $.92(.91) *$ & $1.86(1.25)$ & $\mathbf{2 . 7 8}(\mathbf{1 . 7 4})$ \\
\hline $.61(.49)$ & $1.64(1.07)$ & $\mathbf{2 . 6 1}(\mathbf{1 . 5 5})$ \\
\hline
\end{tabular}

The significant differences between same condition (desktop ILL $->$ mobile ILL) in both environments is indicated on the table. Chi-Square Test: $* \mathrm{p}<.05, *$ p $<.01$, * $* \mathrm{p}<.001$, 粎p $<.0001$

Table 2: Statistical difference between conditions

\begin{tabular}{|c|c|c|}
\hline & \multicolumn{2}{|c|}{ ISL } \\
\hline Measure & Desktop & Mobile \\
\hline NumPage & 1.41 & 0.63 \\
\hline NumExam & 3.85 & $7.00 *$ \\
\hline DRS & 3.77 & 1.21 \\
\hline DRH & 16.08粶* & - \\
\hline ROA & 2.60 & 4.00 \\
\hline DRT & 65.11 米光 & 37.40粶* \\
\hline TTotal & 39.20 米㫧 & 333.67 米粶 \\
\hline TRele & 37.62 米永 & 44.45䊑粶 \\
\hline
\end{tabular}

The significant differences across the conditions are indicated. Chi-Square Test: $* \mathrm{p}<.05, * \mathrm{p}<.01, * * \mathrm{p}<.001$, **** $\mathrm{p}<.0001$

search results were present in both environments. When comparing between both environments, while more search results scrolled into view on desktop, the depth of search results saved as relevant by users was lower on desktop compared to on mobile when there was only 1 relevant result (ILL condition). This suggest that users were less likely to save relevant documents (less than 1) when the number of relevant search results were too few. Conversely, it also suggested that mobile users were more tolerant of non-relevant search results for ILL condition by saving some non-relevant (more than 1) search results as relevant. On mobile, we can only display 2-3 search results due to the smaller screen. We observed that displaying 5 relevant search results did not increase the number of documents clicked by users. In fact, click-through rate were lower when more snippets were present from 3 to 5 relevant search results. We speculated that by having enough number of relevant search results, users were able to determine any of the search results were relevant by just viewing the snippets. This is, however, different on desktop, the number of examined documents increased with increasing number of relevant search results. In terms of statistical significance, the varying information scent level influenced search behavior in terms of DRT, TTotal and TRele in general, DRH on desktop and NumExam on mobile.

\subsection{Future plan}

We have identified a correlation between ISL and search behavior. In future work, we intend to study if Information Scent also affect query reformulations in different environments. Given that we also collected typing behavior, we will like to analyze if query reformulations behavior is influenced by typing fluency (that is if the ease of typing on query refor- mulations). We will analyze if Information Scent influenced query reformulations in terms of session-level, task-level or query-level differences [2].

Additionally, we will also like to conduct a follow-up experiment to understand if varying snippet length on both mobile and desktop crowd-source experiments have an influence on search behavior. We plan to combine both typing behavior and snippet length to build new user models based on Information Foraging Theory to predict web search behavior in different environments. Keystroke biometrics are already used in other areas such as for user authentication. ${ }^{3}$ It is plausible that user characteristics such as their search experience and even topic familiarity may be informed by their typing behaviors, such as typing speed, rhythm and pauses.

\section{REFERENCES}

[1] Cutrell, E., AND Guan, Z. What Are You Looking For?: An Eye-tracking Study of Information Usage in Web Search. In Proceedings of SIGCHI (2007), pp. $407-416$.

[2] JiAng, J., AND Ni, C. What Affects Word Changes in Query Reformulation During a Task-based Search Session? In Proceedings of CHIIR (2016), pp. 111-120.

[3] Oppenheimer, D. M. The secret life of fluency. Trends in Cognitive Sciences 12, 6 (2008), 237-241.

[4] Pirolli, P., And Card, S. Information Foraging. Psychological Review 106, 4 (1999), 643-675.

[5] Song, Y., Ma, H., Wang, H., And Wang, K. Exploring and exploiting user search behavior on mobile and tablet devices to improve search relevance. In Proceedings of $W W W$ (2013), pp. 1201-1212.

[6] Tombros, T., And Crestani, F. Users' perception of relevance of spoken documents. JASIST 51, 10 (2000), 929-939.

[7] White, R., ANd Drucker, S. M. Investigating Behavioral Variability in Web Search. In Proceedings of $W W W$ (2007), pp. 21-30.

[8] Wu, W.-C., AND Kelly, D. Online search stopping behaviors: An investigation of query abandonment and task stopping. 77th ASISET Annual Meeting (2014).

[9] Wu, W.-C., Kelly, D., And Sud, A. Using Information Scent and Need for Cognition to Understand Online Search Behavior. In Proceedings of SIGIR (2014), pp. 557-566.

\footnotetext{
${ }^{3}$ http://www.keytrac.net/
} 